\title{
La protection du bien-être animal en droit communautaire: Avancées, limites et propositions futures
}

\section{Sabine Brels ${ }^{1}$.}

L'Union européenne (UE) accorde une place de plus en plus importante à la protection du bien-être animal. Toutefois, malgré des avancées notables et des normes élevées sur la scène internationale, cette protection comporte encore d'importantes limites et des lacunes persistantes. L'évolution de cette protection peut être évaluée en regard des deux catégories de sources du droit communautaire ${ }^{2}$ : d'abord au sein des traités de l'Union européenne (en droit communautaire primaire), ensuite au sein des directives et règlements (en droit communautaire dérivé). Si la protection du bien-être animal a d'abord figuré en annexe des traités de I'UE avant de devenir un objectif principal au sein des nouveaux traités (1), cette protection se voulant élevée comporte toujours des limites et lacunes de taille (2).

\section{1- Le bien-être animal en droit primaire: un objectif annexe devenu principal dans les traités de I'UE}

Au niveau communautaire, l'apparition du concept de "bien-être animal" est intrinsèquement liée à la constitution de l'Union européenne. En effet, ce concept a été consacré pour la première fois en 1992 au sein d'une déclaration annexée au Traité de Maastricht établissant I'UE ${ }^{3}$. Bien que pionnière, cette consécration du bien-être animal en tant que nouvelle exigence à prendre en compte en droit communautaire, n'était alors que d'une déclaration de bonne volonté sans valeur contraignante ${ }^{4}$. Toutefois, une première étape vers le renforcement de cette exigence a été franchi cinq ans plus tard

\footnotetext{
1 Doctorante en droit à l'Université Laval (Québec), spécialiste en droit animal, titulaire de la bourse d'études supérieures du Canada Vanier

${ }^{2}$ Le "droit communautaire" fait référence au droit de l'Union européenne.

${ }^{3}$ Déclaration relative à la protection des animaux $\left(n^{\circ} 24\right)$ annexée au Traité sur l'Union européenne, Maastricht, J.O. C 191 du 29 juillet 1992.

${ }^{4}$ Voir Suzanne ANTOINE, Le droit de l'animal, Legis-France, 2007, citée dans J.-P. MARGUÉNAUD, "La promotion des animaux au rang d'êtres sensibles dans le Traité de Lisbonne", RSDA 2/2009, p.14.
} 
avec l'adoption du Protocole annexé au Traité d'Amsterdam en $1997^{5}$. Bien que ce protocole présente le mérite d'ajouter le bien-être animal aux objectifs de I'UE, celui-ci demeure un objectif "annexe" revêtant seulement une valeur secondaire ${ }^{6}$. Cette situation a changé depuis les derniers traités puisque le bien-être animal figure désormais au cœur même des objectifs européens comme un nouveau principe général et constitutionnel de droit communautaire ${ }^{7}$. En effet, celui-ci a d'abord été intégré à l'article III-121 du Traité établissant une constitution pour l'Europe de 2004, et plus récemment à l'article13 du Traité de Lisbonne de 2008. La formulation de ces articles rassemble les éléments préfigurant dans la déclaration de 1992 et du Protocole de 1997 en ces termes:

"Lorsqu'ils formulent et mettent en œuvre la politique de I'Union dans les domaines de l'agriculture, de la pêche, des transports, du marché intérieur, de la recherche et développement technologique et de l'espace, I'Union et les États membres tiennent pleinement compte des exigences du bien-être des animaux en tant qu'êtres sensibles, tout en respectant les dispositions législatives ou administratives et les usages des États membres, notamment en matière de rites religieux, de traditions culturelles et de patrimoines régionaux." 8

En résumé, le bien-être animal serait une "exigence à prendre en compte" dans le cadre de certaines politiques communautaires, notamment en matière d'élevage d'animaux terrestres et aquatiques (agriculture et pêche), de transports, de commerce (marché intérieur) et d'expérimentation

\footnotetext{
${ }^{5}$ Protocole sur la protection et le bien-être des animaux $\left(n^{\circ} 10\right)$ annexé au Traité d'Amsterdam modifiant le traité sur l'Union européenne, les traités instituant les Communautés européennes et certains actes connexes, Amsterdam, J.O. C 340 du 10.11.1997.

${ }^{6}$ La valeur secondaire de l'exigence de bien-être animal a été notamment confirmée dans la décision "Jippes" de 2001. Selon l'interprétation de la CJCE, le bien-être animal n'est pas, tel que consacré par le Protocole, un principe général de droit communautaire. De plus, cette exigence est inférieure aux impératifs d'intérêt général comme la sécurité sanitaire et surtout la rentabilité économique des éleveurs européens. Dans cette décision, la Cour a validé les mesures d'interdiction de vaccination préventive et d'abattage systématique du bétail afin d'éradiquer l'épidémie de fièvre aphteuse. Voir CJCE, H.Jippes, Aff. C-189/01, [ECR I-5689], 12 juillet 2001, Rec. 2001 p. I-05689.

${ }^{7}$ Voir S.BRELS, El bienestar de los animales: un nuevo principio general y constitucional de Derecho comunitario, Derecho animal, Mayo 2012.

${ }^{8}$ Art. III-121 du Traité établissant une Constitution pour l'Europe, J.O.U.E. C 310/55 du 16.12.2004 et Art.13 du Traité sur l'Union européenne et sur le fonctionnement de l'Union européenne (version consolidée), J.O. C 115/01 du 09.05.2008.
} 
(recherche, développement technologique et de l'espace) ${ }^{9}$. Bien que ces traités permettent d'ériger le statut des animaux au rang $d^{\prime \prime}$ "êtres sensibles" ${ }^{10}$, ceux-ci limitent pourtant la prise en compte de leur bien-être aux domaines explicitement prévus. En outre, leur protection est d'autant plus limitée qu'elle doit se plier à la suprématie des intérêts économiques, culturels et religieux en présence dans I'UE.

L'objectif économique, à la base même de la construction de la Communauté économique européenne en 1957, reste très présent dans le cadre de I'UE depuis 1992, même si cette institution a pris une tournure plus politique ${ }^{11}$. C'est pourquoi, bien que I'UE soit prête à faire certaines concessions pour améliorer le sort des animaux d'élevage et d'expérimentation, ces améliorations ne doivent pas pour autant nuire à la compétitivité des producteurs européens ${ }^{12}$. Quant aux exceptions culturelles et religieuses mentionnées dans la formule énoncée, il est possible de fournir une illustration pour chacune d'entre elles. Concernant les "rites religieux", il est possible de citer le cas des abattages rituels dérogatoirement autorisés, alors même que l'étourdissement préalable est normalement obligatoire depuis 1974 , et désormais en vertu de la directive sur l'abattage et la mise à mort des animaux de $1993^{13}$. En ce qui concerne les "traditions culturelles", citons l'exemple la tauromachie impliquant généralement une mise à mort douloureuse, laquelle est en principe interdite par ces mêmes dispositions ${ }^{14}$. Notons que la tauromachie figure également au sein du patrimoine culturel immatériel français depuis 2011, comme le foie gras depuis 2005, ceux-ci relevant ainsi de l'exception

\footnotetext{
${ }^{9}$ Le Professeur Marguénaud précise que "I'adjonction du développement technologique et de l'espace" aux domaines prévus par le Protocole de 1997 "semble pouvoir s'expliquer par le souci de soumettre aux exigences du respect du bien-être des animaux en tant qu'êtres sensibles toutes les expérimentations qu'ils subissent à quelque titre que ce soit et où que ce soit". Op.cit., note 4, p.18.

${ }^{10} / d$., p.16. Puisque les animaux sont désormais reconnus en tant qu'être sensibles, il ne serait plus possible "de les assimiler purement et simplement à des produits asservis aux exigences du marché".

${ }^{11}$ Rappelons que le Traité de Rome en 1957 a permis l'établissement de la CEE, laquelle a été remplacée en 1992 par l'UE dans le cadre du traité de Maastricht.

12 Cette idée est rappelée dans le cadre de la Stratégie 2012-2015, Section 3.3 [COM(2012) 6 final/2]: "Des conditions de concurrence équitables au niveau mondial dans le domaine du bien-être animal sont importantes pour assurer la compétitivité internationale des exploitants de I'Union".

${ }^{13}$ Voir l'Art.5 (2) de la Directive 93/119/CE du Conseil, du 22 décembre 1993, sur la protection des animaux au moment de leur abattage ou de leur mise à mort, J.O. L 340 du 31.12.1993.

${ }^{14}$ Précisions qu'en vertu du principe de subsidiarité, la réglementation de ce type de manifestations comme les spectacles avec animaux relèvent de la compétence de chaque Etat et ne sont pas du ressort de l'UE.
} 
prévue pour les "patrimoines régionaux". En ce sens, bien que la pratique du gavage soit en principe interdite par la directive de 1998 sur les élevages, ainsi que par la Convention européenne de $1976^{15}$, le foie gras français est exempté puisqu'il fait légalement "partie du patrimoine culturel et gastronomique protégé en France" ${ }^{16}$.

Au-delà des traités de l'UE, de nombreuses directives et règlements communautaires ont été adoptés afin de protéger le bien-être des animaux, ou plutôt tenter d'éviter le mal-être de ces derniers autant que possible.

\section{2- Le bien-être animal en droit dérivé: une protection élevée mais limitée dans les directives et règlements de I'UE}

La première directive communautaire adoptée afin d'éviter aux animaux "tout traitement cruel ou toute souffrance inutile" concernait l'obligation d'étourdissement préalable à la mise à mort en 1974 (sauf pour les abattages rituels) ${ }^{17}$. Depuis, des directives-cadres ont été adoptées dans le cadre de l'abattage, de l'élevage et de l'expérimentation des animaux, ainsi que des règlements sur le transport et le transit des animaux de compagnie. Des instruments spécifiques ont également été adopté concernant l'élevage de certaines espèces (veaux, porcs, poulets de chair et poules pondeuses) et une réglementation sur les produits "bio" vise à renforcer les normes de protection dans les élevages ${ }^{18}$. Sur le plan international, des embargos ont été adoptés sur les peaux de bébés phoques (1983), les fourrures issues de piège à mâchoires (1991), les fourrures de chiens et chats (2007), et les produits dérivés du phoque $(2009)^{19}$. Ainsi, les normes communautaires sur le bien-être animal se veulent

\footnotetext{
${ }^{15}$ Voir l'Annexe, al.14, de la Directive 98/58/CE du 20 juillet 1998 concernant la protection des animaux dans les élevages, J.O. L 221, 08/08/1998 et l'Art. 6 de la Convention européenne de 1976 sur la protection des animaux dans les élevages Voir le Préambule (2è considérant) et l'Art.4 de la Directive 74/577/CEE du Conseil, du 18 novembre 1974, relative à l'étourdissement des animaux avant leur abattage, J.O. L 316 du 26.11.1974.

${ }^{16}$ Amendement $n^{\circ} 1001$ à la Loi d'orientation agricole ( $n^{\circ}$ 2341) du 5 octobre 2005 ajoutant l'Art. L. 654-27-1 au Code rural.

${ }^{17}$ Voir l'Art.4 de la Directive 74/577/CEE du Conseil relative à l'étourdissement des animaux avant leur abattage, J.O. L 316 du 26.11.1974.

${ }^{18}$ Voir S.BRELS, "Les limites de la réglementation "bio" en matière de protection du bien-être animal", Revue de la LFDA, n75, Octobre 2012.

${ }^{19}$ Voir la liste des instruments (inventaire personnel), en ligne:

http://www.animallaw.info/nonus/articles/art_pdf/aranimalwelfareeuropean.pdf.
} 
garantes d'une protection élevée sur la scène internationale. En tant qu'organisation supranationale, I'UE souhaite continuer à assurer un certain leadership en la matière à l'échelle mondiale. Celle-ci entend ainsi renforcer sa coopération avec les organisations intergouvernementales jouant un rôle clé en matière de commerce et d'agriculture, à savoir l'Organisation mondiale du commerce (OMC) et I'Organisation des Nations unies pour l'alimentation et l'agriculture (FAO). II s'agit aussi de soutenir les initiatives protectrices sein du Conseil de l'Europe et de I'Organisation mondiale de la santé animale (OIE) en jouant un rôle actif et proactif au sein de ces organisations pionnières pour la protection du bien-être animal aux niveaux européen et mondial ${ }^{20}$.

Parmi les instruments communautaires adoptés pour protéger le bien-être des animaux, l'avancée la plus notable est sans doute l'interdiction d'expérimentation des produits cosmétiques et la mise sur le marché des produits testés sur les animaux pour $2013^{21}$. D'autres avancées revendiquées comme importantes sont néanmoins plus limitées. Par exemple, I'obligation de remplacement, au 1er janvier 2012, des cages en batterie traditionnelles par des cages aménagées pour les poules pondeuses, n'accorde en fait que l'équivalent d'une carte postale comme espace supplémentaire à la feuille de papier A4 dont disposait chaque poule dans les cages précédentes ${ }^{22}$. Comme pour les autres espèces, les normes communautaires permettent ainsi d'augmenter quelque peu l'espace alloué aux individus détenus, mais l'état de confinement reste malgré tout "intensif", à proprement parler, dans la plupart des élevages industriels ${ }^{23}$. En ce sens, les instruments communautaires sur le bien-être animal ne manquent pas de préciser qu'ils visent seulement à établir des "normes minimales" de protection. Somme toute, ces normes ne semblent même pas minimales lorsqu'elles autorisent, par exemple, les mutilations courantes des animaux d'élevage sans anesthésie ni analgésie systématique. Pratiquées "à vif", celles-ci concernent notamment l'édentage ainsi que la castration et la coupe de la queue des

\footnotetext{
${ }^{20}$ Le Plan d'action 2006-2010 et la Stratégie 2012-2015 sur le bien-être animal confirment la volonté de leadership de I'UE au plan international.

${ }^{21}$ La fin de toutes les expérimentations est prévue pour le 11 mars 2013. Voir l'Art.18 du Règlement (CE) $n^{\circ} 1223 / 2009$ du Parlement européen et du Conseil du 30 novembre 2009 relatif aux produits cosmétiques, J.O. L 342 du 22.12.2009.

${ }^{22}$ Art.5-2 de la Directive 1999/74/CE du Conseil du 19 juillet 1999 établissant les normes minimales relatives à la protection des poules pondeuses, J.O. L 203 du 3.8.1999. L'espace réglementaire est passé de 550 à $750 \mathrm{~cm} 2$ d'espace disponible par poule (Art. 5-1 (1) et 6-1 (a)).

${ }^{23}$ Voir le site de la PMAF, "Interdiction des cages conventionnelles en 2012": http://pmaf.org/s-informer/noscampagnes/poules-pondeuses.html.
} 
porcelets (avant sept jours), l'écornage des bovins ou encore la coupe du bec des poules ${ }^{24}$. L'argument pratique pour justifier de telles pratiques est d'éviter que les animaux ne se blessent entre eux ${ }^{25}$. À cela, il est possible de répondre que ces blessures n'arriveraient pas si les animaux avaient plus d'espace. Quant à l'argument économique, celui-ci vient, de toute évidence, expliquer pourquoi les anesthésies et analgésies coûteuses ne sont pas pratiquées systématiquement.

Sur les atteintes volontaires à l'intégrité physique des animaux, il est possible de relever que, contrairement aux animaux d'élevage, les animaux de compagnie sont protégés des interventions noncuratives par la Convention européenne de 1987. Les pratiques interdites dans le cadre de celle-ci concernent notamment la coupe de la queue et des oreilles, la section des cordes vocales, ainsi que l'ablation des dents ou des griffes (concernant notamment les chiens et les chats) ${ }^{26}$. Il est possible de regretter que ces dispositions n'aient pas été intégrées en droit communautaire, alors que, dans le cadre des autres conventions européennes sur l'abattage, l'élevage et l'expérimentation, I'UE est même allée au-delà des dispositions prévues par le Conseil de l'Europe ${ }^{27}$. Bien que certains pays membres de l'UE ait ratifié la convention européenne sur la protection des animaux de compagnie, l'absence de protection de ces derniers constitue une lacune importante en droit communautaire ${ }^{28}$. En effet, seul un règlement sur les mouvements non-commerciaux des animaux de compagnie a été

\footnotetext{
${ }^{24}$ Directives porcs (Directive 2008/120/CE), veaux (Directive du Conseil 2008/119/CE) et poules pondeuses (Directive du Conseil 1999/74/EC).

${ }^{25}$ Par exemple la directive sur les porcs autorise la "section partielle de la queue et la réduction des coins", en principe seulement "lorsqu'il existe des preuves que des blessures causées aux mamelles des truies ou aux oreilles ou aux queues d'autres porcs ont eu lieu" (Annexe I, Chap. I, al.8).

${ }^{26}$ Art. 10 de Convention européenne pour la protection des animaux de compagnie ( $\left.n^{\circ} 123\right)$, signée le 13.11.1987, entrée en vigueur le 1.05.1992.

${ }^{27}$ Par exemple en matière d'élevage, la Directive 98/58/EC contient des normes plus strictes que la Convention européenne sur la protection des animaux dans les élevages et en matière d'expérimentation, la Directive 2010/63/UE va au-delà de la protection des vertébrés dans la Convention européenne sur la protection des animaux vertébrés utilisés à des fins expérimentales ou à d'autres fins scientifiques en protégeant certains invertébrés (comme les céphalopodes) et en étendant sa protection aux stades précoces de développement (formes larvaires, fœtus et embryons).

${ }^{28}$ Les pays membres de I'UE (et du Conseil de l'Europe), qui l'ont ratifié sont: I'Allemagne, la Belgique, le Danemark, la France, la Finlande, la Lettonie, le Luxembourg, le Portugal et la République Tchèque. En plus de ces pays, l'Azerbaïdjan a également ratifié cet convention.
} 
adopté dans le cadre de I'UE, celui-ci visant uniquement le respect des formalités sanitaires et non le bien-être des animaux ${ }^{29}$.

Si un instrument communautaire en venait à être adopté, celui-ci pourrait par exemple exiger un certificat d'aptitude afin d'adopter un animal de compagnie, de sorte à s'assurer que la personne responsable sera, notamment, en mesure d'en prendre soin et de répondre à ses besoins physiologiques et comportementaux spécifiques ${ }^{30}$. En effet, les besoins des animaux diffèrent d'une espèce à l'autre et ceux-ci ne sont pas toujours bien connus des adoptants. De plus, ceux-ci peuvent encore décider d'adopter un animal sur un "coup de tête" sans aucune notion des responsabilités impliquées pour garantir leur bien-être. Le droit devrait ainsi permettre d'empêcher les adoptions "irréfléchies" afin que les animaux ne soient pas abandonnés à eux-mêmes dans les foyers, maltraités, négligés ou encore abandonnés lorsque l'adoptant ne désire plus garder son animal. Les animaux ne sont pas des marchandises que l'on achète et qu'on jette. En tant qu'"êtres sensibles" reconnus juridiquement comme tels, ils méritent notre attention et tout adoptant devrait pouvoir s'engager contractuellement à s'occuper de son animal pour toute la vie de ce dernier. Car le caractère "sensible" de l'animal est à prendre ici dans double sens. L'animal est capable de souffrir, mais il peut aussi éprouver des sentiments. C'est pourquoi le lien d'affection qui se tisse entre un animal et son adoptant ne devrait pas pouvoir être brisé impunément à volonté. Ce lien particulier est même reconnu par la Convention européenne de 1987 qui décourage également le fait de détenir des animaux sauvages en tant qu'animaux de compagnie ${ }^{31}$. En effet, le phénomène de mode actuel concernant les "nouveaux animaux de compagnie" (dits "NAC") pose de nombreux problèmes quant à la garde des animaux, voire des problèmes de sécurité lorsque ceux-ci sont relâchés dans la nature ${ }^{32}$. En ce qui concerne les animaux sauvages en captivité, il convient de préciser la directive communautaire sur la protection des animaux dans les zoos a pour objectif la conservation de la

\footnotetext{
${ }^{29}$ Par exemple, le Règlement (CE) $n^{\circ} 998 / 2003$ exige un certificat de santé valide lors du transit des animaux de compagnie dans I'UE (Art. 8-2).

${ }^{30}$ Ces termes et ces obligations reviennent souvent dans les différents instruments communautaires liés à la protection du bien-être des animaux.

${ }^{31}$ Voir les alinéas 3, 7 et 9 de la Convention européenne pour la protection des animaux de compagnie $\left(n^{\circ} 123\right)$.

32 M. BATISTE, "Les nouveaux animaux de compagnie: un engouement, un trafic", dans F. BURGAT (dir.), L'animal dans nos sociétés, p.82-83.
} 
biodiversité et non la protection du bien-être des animaux captifs ${ }^{33}$. Il en va de même du règlement sur la protection des cétacés dans le cadre des pêcheries, qui vise à conserver la population menacée des marsouins en mer baltique, mais non pas l'interdiction des méthodes de pêches cruelles auxquels ils sont confrontés ${ }^{34}$. Ces méthodes, comme certains filets de pêche à l'origine de nombreuses "prises accidentelles" causant la mort de milliers de dauphins ${ }^{35}$. En ce sens, l'absence de protection du bienêtre des animaux sauvages, tant en liberté qu'en captivité, constituent des lacunes supplémentaires en droit communautaire.

Concernant les animaux sauvages en liberté, l'UE pourrait également imposer des normes minimales sur la chasse, la pêche et le piégeage, notamment afin d'interdire les méthodes les plus cruelles. Pour ce qui est des animaux sauvages en captivité, le droit communautaire pourrait sans doute, dans le cadre de son objectif de renforcement de la protection du bien-être animal, réglementer les conditions de détention des animaux dans les zoos et les parcs aquatiques, sans oublier leurs méthodes de dressage (ou "domptage") dans les cirques ${ }^{36}$. Quant aux différentes manifestations publiques comme les exhibitions, compétitions et spectacles avec animaux, notons que le Conseil de l'Europe avait l'intention de rédiger un projet de convention à ce sujet, lequel a été avorté suite à la suppression du Comité permanent sur la protection des animaux en 1987. Cette suppression non remise en cause peut être regrettée en regard du travail considérable réalisé par ce Comité d'experts afin de construire les fondations de la protection européenne du bien-être animal ${ }^{37}$. Néanmoins, notons que le Conseil de l'Europe n'a pas arrêté sa mission protectrice puisqu'il continue a adopter des recommandations

\footnotetext{
${ }^{33}$ Art.1 de la Directive 1999/22/CE du Conseil, du 29 mars 1999, relative à la détention d'animaux sauvages dans un environnement zoologique, J.O. L 094 du 09.04.1999.

${ }^{34}$ Art.1 du Règlement (CE) $n^{\circ}$ 812/2004 du Conseil du 26 avril 2004 établissant des mesures relatives aux captures accidentelles de cétacés dans les pêcheries et modifiant le règlement (CE) nº 88/98, J.O. L 150 du 30.04.2004.

${ }^{35}$ En plus des dauphins, les baleines, tortues et oiseaux sont pris dans les filets maillants et les filets perdus qui dérivent en mer: "On dénombre chaque année plus d'un million de ces morts non intentionnelles". Voir J.B. JEANGÈNE VILMER, Éthique animale, PUF, 2008, p.254.

${ }^{36}$ Voir V. DERIABKINE, "L'expérience d'un dompteur" ainsi que J.-C. NOUET et S. NE, "La condition animale dans les cirques", dans F. BURGAT, L'animal dans nos sociétés, Paris, Documentation française, 2004, pp.90-92.

${ }^{37}$ E. AUSEMS, "Le Conseil de l'Europe et la protection des animaux", dans BROOM, D. M. et al, Bien-être animal, éditions du Conseil de l'Europe, 2006, p.270. Bien qu'une recommandation de 1999 demandait que le Comité puisse "continuer à développer les activités" pour protéger le bien-être des animaux, le Comité des Ministres du Conseil de l'Europe a refusé de la suivre.
} 
pour le bien-être de différentes espèces, dont plusieurs n'ont pas non plus été intégrées par l'Union européenne. Citons notamment les différentes recommandations sur les animaux élevés pour leurs fourrures, les palmipèdes à foie gras ou encore les poissons d'élevage ${ }^{38}$.

\section{Conclusion sur les avancées futures}

Depuis son plan d'action 2006-2010, et désormais dans sa stratégie 2012-2015 sur le bien-être des animaux, I'Union européenne prévoit de renforcer ses mesures de protection et de combler les lacunes existantes. En ce sens, il s'agirait notamment d'adopter des instruments pour les espèces qui ne disposent encore d'aucune protection communautaire ${ }^{39}$.

En particulier, I'UE prévoit de trouver des alternatives afin d'interdire la castration des porcs pour 2018 et les cases de gestation pour les truies en 2019. De plus, une législation-cadre permettant de codifier l'ensemble des instruments communautaires sur la protection du bien-être animal devrait également voir le jour dans les prochaines années ${ }^{40}$.

Pour l'instant, il est possible d'avancer que, bien qu'avant-gardiste dans certains domaines, la réglementation communautaire n'en demeure pas moins timide sous d'autres aspects. En effet, celleci est tiraillée par la contradiction interne aux divergences protectionnistes et non-protectionnistes de ses états membres ${ }^{41}$. Alors que certains pays poussent en faveur d'une protection accrue et d'un leadership affirmé en matière de protection du bien-être animal sur la scène internationale, d'autres visent à freiner cette protection afin de protéger leurs intérêts nationaux particuliers. Cette schizophrénie communautaire sur la protection du bien-être animal résulte en une protection qui

\footnotetext{
${ }^{38}$ Recommandations sur les palmipèdes à foie-gras et les animaux à fourrure du 22 juin 1999 et celle sur les poissons d'élevage de 2005.

${ }^{39}$ Plan d'action 2006-2010 [J.O. C 49, 28.02.2006] et la Stratégie 2012-2015 [COM(2012) 6 final/2] sur la protection et le bien-être des animaux.

40 A.GAVINELLI, "The enforcement preparation of the EU Commission after the Laying Hens experience", Enforcement of European Animal Welfare Related Legislation, Eurogroup for Animals Conference, Brussels, 13.06.2012. Cf. "Actions prévues", Annexe de la Stratégie 2012-2015.

${ }^{41}$ Parmi les anciens pays de I'UE, les Etats pouvant être qualifiés de "protectionnistes" (ou moteur de protection) sont notamment l'Angleterre et l'Allemagne; quant aux pays "non-protectionnistes" (plus réticents envers cette protection), il s'agit par exemple de la France et l'Espagne. Voir N. MELIK, "La France face au "droit des animaux"" et M.R., GAMERO, "L'Espagne, un pays non-protectionniste", dans BROOM, D. M. et al, Bien-être animal, éditions du Conseil de l'Europe, 2006, pp. 227-248.
} 
semble à la fois enthousiaste et pleine de bonne volonté, mais finalement très relative et surtout assez hypocrite dans le fond. En effet, comment peut-on parler de normes protectrices lorsque le confinement intensif, l'égorgement à mort ou les mutilations à vifs des animaux sont cautionnées par la législation-même censée garantir leur bien-être?

Derrière des apparences parfois très protectrices réitérant de nombreuses fois l'objectif de protéger le bien-être des animaux en tant qu'être sensibles, notamment à travers l'obligation de leur éviter toute douleur et souffrance, on se rend compte que certaines exceptions ou dispositions particulières viennent annihiler, sinon amoindrir considérablement, la portée protectrice de ces instruments. C'est pourquoi on peut confirmer que, en droit en général, et en matière de protection du bien-être animal en particulier: le diable se cache certainement dans les détails!

En résumé, bien que la protection allouée par l'Union européenne au bien-être des animaux soit élevée sur la scène internationale, celle-ci comporte encore de nombreuses failles. Néanmoins, celle-ci permet déjà de fournir un exemple à la fois opératoire et obligatoire de protection du bien-être animal au sein d'une organisation supranationale. Celle-ci permet également de "tirer vers le haut" les étatsmembres les plus réticents à faire des concessions en faveur de cette protection. En effet, certaines avancées n'auraient jamais eu lieu dans les pays dits "non-protectionnistes" si les normes de protection de I'UE n'avaient pas vu le jour. Enfin, bien que certaines interdictions commencent à poindre, par exemple concernant les tests des produits cosmétiques et les cages en batterie traditionnelles, l'approche de l'UE n'est définitivement pas "abolitionniste" puisqu'elle ne remet pas en question le principe même d'utilisation des animaux. Au contraire, celle-ci continue à encourager fermement cette utilisation, notamment dans le cadre de l'élevage et de l'expérimentation, même si elle précise que le bien-être des animaux ne doit pas pour autant être ignoré. En ce sens, si les conditions de vie et de mort des prisonniers sont un peu améliorées, ceux-ci ne sont pas pour autant libérés! 